\title{
Influence of Dipole-Dipole Cross-Relaxation on Spectral Lineshapes of Methyl Protons in Inversion-Recovery Experiments
}

\author{
TaI-Sung LeE AND Lian-Pin Hwang* \\ Department of Chemistry, National Taiwan University, Taipei, Taiwan, Republic of China, and Institute \\ of Atomic and Molecular Sciences, Academia Sinica, Taipei, Taiwan, Republic of China
}

Received January 9, 1990

\begin{abstract}
The effects of dipole-dipole cross-relaxations on the spectral lineshape of the methyl protons have been examined from inversion-recovery measurements. The approach of the state multipole formalism is invoked in the interpretation of relaxation processes and the analyses of lineshapes. Excellent agreements between the observed spectra and the simulation demonstrate that the measurement of spectral lineshapes by inversion recovery provides a convenient method for the study of dipole-dipole cross-relaxation of methyl protons. (c) 1990 Academic Press, Inc.
\end{abstract}

The spectral lineshapes obtained in the inversion-recovery experiment are usually identical to those obtained after a $90^{\circ}$ pulse applied to an initial equilibrium spin system. In a recent study, it was demonstrated theoretically that for quadrupolar nuclei in the slow motional region the effects of the higher-rank multipoles (rank $\geqslant 3$ ) may be generated after the spin inversion in the $180^{\circ}-\tau-\theta$ pulse experiment $(1)$. Although the calculated NMR lineshape is dependent on $\tau$ and $\theta$, the effect is experimentally insignificant.

In the study of methyl dynamics by proton $T_{1}$ measurements, it has been observed that the deviation of the magnetization evolution from exponential behavior is too small to permit one to ascertain the dipole -dipole cross-relaxations (2). The advent of multiple-quantum coherence experiments opens the possibility of selecting the appropriate spin interaction which generates only a certain order of multiple-quantum coherence (3). Thus, several authors have demonstrated that dipole-dipole cross-relaxation may lead to the conversion of single-quantum coherence into multiple-quantum coherence $(4,5)$ and the Zeeman order into three-spin order $(6,7)$. It has then afforded new ways to investigate the dipole-dipole cross-interactions among the methyl protons in liquids. In the work of Böhlen et al., a so-called "pseudo-twodimensional" spectrum has been invoked to demonstrate the effect of dipole-dipole cross-relaxation (6). Wang and Hwang reported the results of a study which utilizes a one-dimensional analogue of the triple-quantum $2 \mathrm{D}$ experiment via longitudinal relaxation to determine the relaxation behavior of three-spin order for the methyl protons in the deoxycholate micelle system (7).

\footnotetext{
* To whom correspondence should be addressed.
} 
It is the purpose of this paper to show that in the usual inversion-recovery experiments the effects of dipole-dipole cross-relaxation may be revealed from the studies of the spectral lineshape patterns. It yields significantly different lineshapes from that obtained after a $90^{\circ}$ pulse experiment on an initially prepared equilibrium spin system. To simplify the calculation of relaxation processes, the state multipole approach $(1,8-10)$ is utilized in the formulation and interpretation of relaxation theory.

\section{THEORY}

The spin-lattice interaction Hamiltonian of this system is defined for the intramolecular dipolar interactions among the three protons of the methyl group by

$$
\mathscr{H}(t)=\sum_{i<j} \epsilon_{i j} \sum_{m}(-1)^{m} T_{2}^{m}(i, j) Y_{2}^{-m}\left(\phi_{i j}\right),
$$

where $\epsilon_{i j} \equiv(\sqrt{6 \pi / 5}) \gamma_{i} \gamma_{j} h / r_{i j}^{3}, T_{2}^{m}(i, j)$ is a spin tensor operator of rank $2, Y_{2}^{m}\left(\phi_{i j}\right)$ is a spherical harmonic function of rank $2(11), \gamma_{i}$ is the gyromagnetic ratio of species $i$ of the methyl protons, and $r_{i j}$ is the distance between protons $i$ and $j$ in the methyl group.

The interferences between the internal rotation of the methyl group and the overall reorientation of the whole molecules are included to facilitate the calculation of the auto- and cross-correlation spectral densities. The dynamics of the molecule is described by an isotropic slow reorientation of the whole molecule and the fast internal motions for the methyl group of interest. With consideration of the auto- and crosscorrelation of dipolar relaxations (2) among the three protons, the auto- and crossspectral density functions used in the calculation of the relaxation matrix elements are given by

$$
\begin{aligned}
J_{i j k l}^{m n}(\omega) & =\epsilon_{i j} \epsilon_{k l}(-1)^{n} \int_{0}^{\infty}\left\langle Y_{2}^{m}\left(\phi_{i j}(t)\right) Y_{2}^{-n}\left(\phi_{k l}(t+\tau)\right)\right\rangle \cos \omega \tau d \tau \\
& =\delta_{m-n} \frac{3 \gamma^{4} \hbar^{2}}{10 r^{6}}\left\{\frac{1}{4} \frac{6 D}{(6 D)^{2}+\omega^{2}}+\frac{3}{4} \cos \left(\Omega_{i j k l}\right) \frac{\left(6 D+4 D_{i}\right)}{\left[\left(6 D+4 D_{i}\right)^{2}+\omega^{2}\right]}\right\},
\end{aligned}
$$

where $J_{a}(\omega) \equiv J_{i j k l}^{m n}(\omega)$ with $\cos \left(\Omega_{i j k l}\right)=1$ for $r_{i j}=r_{k l}$ and $J_{c}(\omega) \equiv J_{i j k l}^{m n}(\omega)$ with $\cos \left(\Omega_{i j k l}\right)=-\frac{1}{2}$ for $r_{i j} \neq r_{k l}$ to account for the auto- and cross-relaxations, respectively. Here, the distance between protons in the methyl group is denoted by $r(r=1.838$ $\AA$ ). It is assumed that the overall reorientational motion of the molecule has a correlation time $\tau_{0} \equiv 1 / 6 \mathrm{D}$, the internal rotation of the methyl group has a correlation time $\tau_{i} \equiv 1 / 4 D_{i}$, and the $z$ axis of the molecular frame is presumably parallel to the $C_{3}$ axis of the methyl group $(2,12)$.

The density matrices for the system of the methyl protons are defined as

$$
\tilde{\rho}=\left[\rho_{11}, \rho_{44}, \rho_{77}, \rho_{88}, \rho_{22}, \rho_{66}, \rho_{33}, \rho_{55}\right]
$$

for longitudinal relaxation and

$$
\tilde{\boldsymbol{\rho}}=\left[\rho_{14}, \rho_{47}, \rho_{78}, \rho_{26}, \rho_{35}\right]
$$

for transverse relaxation. The matrix elements are labeled according to the spin en- 


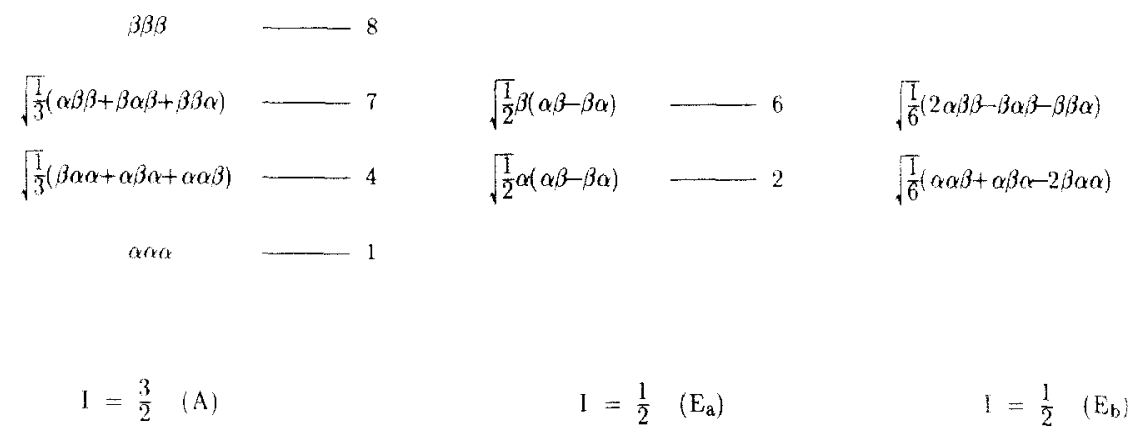

FIG. 1. Energy level diagram and basis functions for the $A_{3}$ spin system. The symmetry of the basis functions is indicated in parentheses.

ergy levels as shown in Fig. 1. The problem may be further simplified by expressing the relaxation equation in a basis where the individual elements of the density matrix transform as components of the full rotation group. The new elements $\sigma_{m}^{k}$ of the density matrix are called statistical tensors or state multipoles $(8,9)$. The new elements can be obtained from the usual density matrix element by

$$
\sigma_{m}^{k}=\sum_{\alpha} \sum_{\alpha^{\prime}} \rho_{\alpha \alpha^{\prime}}(-1)^{I-\alpha}(2 k+1)^{1 / 2}\left(\begin{array}{ccc}
I & I & k \\
\alpha & -\alpha^{\prime} & -m
\end{array}\right),
$$

where $\left(\begin{array}{ccc}i & j & k \\ m & n & q\end{array}\right)$ is a $3-j$ symbol (13). In this new basis the longitudinal and transverse magnetizations are determined by $\sigma_{0}^{1}$ and $\sigma_{1}^{1}$ ( or $\sigma_{-1}^{1}$ ), respectively, where the superscripts indicate the rank of state multipoles. In the periods of longitudinal evolution and in the final detection period, in which the state multipoles evolve, a second-order perturbation treatment such as the Redfield theory (14) is utilized to account for the relaxation processes of the methyl protons. The evolution of the state multipoles $\sigma_{m}^{k}$ follows the equation of motion

$$
\frac{d}{d t} \sigma_{m}^{k}=-i m \omega_{0} \sigma_{m}^{k}+\sum_{k^{\prime}} \mathbf{R}_{m m}^{k k^{\prime}} \sigma_{m}^{k^{\prime}},
$$

where $\omega_{0}$ is the proton Larmor precession frequency and $\mathbf{R}$ is the relaxation matrix in the representation of the state multipole basis. It may be directly derived by the transformation from the Redfield relaxation matrix for appropriate relaxations in accordance with the interaction Hamiltonian defined in Eq. [1]. The description of the relaxation processes is given in terms of the evolution of the state multipoles following the inversion-recovery pulse sequences. Therefore, in order that the longitudinal components of the state multipoles achieve thermal equilibrium values at infinite time, we make an ad hoc assumption by substituting the difference between $\sigma_{0}^{k}$ and its equilibrium distribution value for $\sigma_{0}^{k}$ in the equations of motion presented in this work. Furthermore it is found that the evolution of the longitudinal/transverse state multipole components conserves parity; e.g., if at initial time the spin system contains nonzero longitudinal/transverse components of odd rank then no even rank longitudinal/transverse components will couple with odd rank during evolu- 
tion. The cquation of motion of the state multipoles of interest in longitudinal relaxation may then be simplified by

$$
\frac{d}{d t}\left(\begin{array}{l}
\sigma_{0}^{1}(A) \\
\sigma_{0}^{3}(A) \\
\sigma_{0}^{1}\left(E_{\mathrm{a}}\right) \\
\sigma_{0}^{1}\left(E_{\mathrm{b}}\right)
\end{array}\right)=\mathbf{R}^{\prime}\left(\begin{array}{l}
\sigma_{0}^{1}(A) \\
\sigma_{0}^{3}(A) \\
\sigma_{0}^{1}\left(E_{\mathrm{a}}\right) \\
\sigma_{0}^{1}\left(E_{\mathrm{b}}\right)
\end{array}\right),
$$

where $\mathbf{R}^{\prime}$ is the relaxation matrix for the longitudinal components of the stage multipole. Its elements are given by

$$
\begin{gathered}
R_{11}^{\prime}=1 / 5\left[-J_{\mathrm{a}}(0)+J_{\mathrm{c}}(0)-10 J_{\mathrm{a}}\left(\omega_{0}\right)-2 J_{\mathrm{c}}\left(\omega_{0}\right)-34 J_{\mathrm{a}}\left(2 \omega_{0}\right)-14 J_{\mathrm{c}}\left(2 \omega_{0}\right)\right] \\
R_{12}^{\prime}=1 / 5\left[3 J_{\mathrm{a}}(0)-3 J_{\mathrm{c}}(0)-5 J_{\mathrm{a}}\left(\omega_{0}\right)-19 J_{\mathrm{c}}\left(\omega_{0}\right)+2 J_{\mathrm{a}}\left(2 \omega_{0}\right)+22 J_{\mathrm{c}}\left(2 \omega_{0}\right)\right] \\
R_{13}^{\prime}=1 / \sqrt{10}\left[J_{\mathrm{a}}(0)-J_{\mathrm{c}}(0)-6 J_{\mathrm{a}}\left(2 \omega_{0}\right)+6 J_{\mathrm{c}}\left(2 \omega_{0}\right)\right] \\
R_{14}^{\prime}=R_{13}^{\prime} \\
R_{22}^{\prime}=1 / 5\left[-9 J_{\mathrm{a}}(0)+9 J_{\mathrm{c}}(0)-30 J_{\mathrm{a}}\left(\omega_{0}\right)-18 J_{\mathrm{c}}\left(\omega_{0}\right)-6 J_{\mathrm{a}}\left(2 \omega_{0}\right)-6 J_{\mathrm{c}}\left(2 \omega_{0}\right)\right] \\
R_{23}^{\prime}=1 / \sqrt{10}\left[-3 J_{\mathrm{a}}(0)+3 J_{\mathrm{c}}(0)+5 J_{\mathrm{a}}\left(\omega_{0}\right)-5 J_{\mathrm{c}}\left(\omega_{0}\right)-2 J_{\mathrm{a}}\left(2 \omega_{0}\right)+2 J_{\mathrm{c}}\left(2 \omega_{0}\right)\right] \\
R_{24}^{\prime}=R_{23}^{\prime} \\
R_{33}^{\prime}=\left[-J_{\mathrm{a}}(0)+J_{\mathrm{c}}(0)-2 J_{\mathrm{a}}\left(\omega_{0}\right)+2 J_{\mathrm{c}}\left(2 \omega_{0}\right)-2 J_{\mathrm{a}}\left(2 \omega_{0}\right)+2 J_{\mathrm{c}}\left(2 \omega_{0}\right)\right] \\
R_{34}^{\prime}=0
\end{gathered}
$$

and

$$
R_{44}^{\prime}=R_{33}^{\prime},
$$

where $A, E_{\mathrm{a}}$ and $E_{\mathrm{b}}$ expressed in the parentheses of state multipoles are the irreducible representations of $C_{3 v}$ symmetry, corresponding to the quartet and two doubly degenerate spin states depicted in Fig. 1. It should be noted that all the relaxation matrices shown in this work are real and symmetric. The state multipole with rank three $\sigma_{0}^{3}$ may be rewritten into operator formalism as $\left[5 I_{z}^{j} I_{z}^{k} I_{z}^{l}-I_{z}^{j}\left(\mathbf{I}^{k} \cdot \mathbf{I}^{l}\right)-I_{z}^{k}\left(\mathbf{I}^{l} \cdot \mathbf{I}^{j}\right)\right.$ $\left.-I_{z}^{l}\left(\mathbf{I}^{j} \cdot \mathbf{I}^{k}\right)\right]$ where $I_{z}^{j} I_{z}^{k} I_{z}^{l}$ is defined as the three-spin order operator for spins $j, k$, and $l(7,15)$. It can be shown that in the absence of cross-interaction there is no relaxation for the state multipole with rank three.The detailed relaxation behavior was studies previously for the methyl protons in the deoxycholate micelle system (7).

It is noted that under a resonant RF pulse the transformation of the state multipole components may be described by the relation (10)

$$
\left(\sigma^{+}\right)_{m}^{k}=\sum_{n} D_{m n}^{k}(\varphi-\pi / 2, \theta, \varphi-\pi / 2)\left(\sigma^{-}\right)_{n}^{k},
$$

where $\theta$ is the RF flip angle and $\varphi$ is the RF phase angle referred to the $x$ axis in the rotating frame, and the superscripts $+/-$ indicate the state multipole components after / before the pulse. Thus, after the $\pi / 2$ monitor pulse $(\varphi=\pi / 2$ and $\theta=\pi / 2)$ and 
in the acquisition period, the state multipoles related to the single-quantum coherences at resonance angular frequency $\omega_{0}$ may be evaluated from

$$
\frac{d}{d t}\left(\begin{array}{c}
\sigma_{1}^{1}(A) \\
\sigma_{1}^{3}(A) \\
\sigma_{1}^{1}\left(E_{\mathrm{a}}\right) \\
\sigma_{1}^{1}\left(E_{\mathrm{b}}\right)
\end{array}\right)=\left(\mathbf{R}-\mathrm{i} \omega_{0} \mathbf{I}\right)\left(\begin{array}{c}
\sigma_{1}^{1}(A) \\
\sigma_{1}^{3}(A) \\
\sigma_{1}^{1}\left(E_{\mathrm{a}}\right) \\
\sigma_{1}^{1}\left(E_{\mathrm{b}}\right)
\end{array}\right),
$$

where $\mathbf{R}$ is the relaxation matrix for tranverse components of the state multipoles. Its elements are defined by

$$
\begin{gathered}
R_{11}=1 / 5\left[-13 J_{\mathrm{a}}(0)-5 J_{\mathrm{c}}(0)-22 J_{\mathrm{a}}\left(\omega_{0}\right)-8 J_{\mathrm{c}}\left(\omega_{0}\right)-10 J_{\mathrm{a}}\left(2 \omega_{0}\right)-2 J_{\mathrm{c}}\left(2 \omega_{0}\right)\right] \\
R_{12}=\sqrt{6} / 5\left[-J_{\mathrm{a}}(0)-5 J_{\mathrm{c}}(0)+J_{\mathrm{a}}\left(\omega_{0}\right)-J_{\mathrm{c}}\left(\omega_{0}\right)+6 J_{\mathrm{c}}\left(2 \omega_{0}\right)\right] \\
R_{13}=\sqrt{2} / \sqrt{5}\left[-J_{\mathrm{a}}(0)+J_{\mathrm{c}}(0)-3 J_{\mathrm{a}}\left(\omega_{0}\right)+3 J_{\mathrm{c}}\left(\omega_{0}\right)\right] \\
R_{14}=-R_{13} \\
R_{22}=1 / 5\left[-12 J_{\mathrm{a}}(0)-23 J_{\mathrm{a}}\left(\omega_{0}\right)-7 J_{\mathrm{c}}\left(\omega_{0}\right)-10 J_{\mathrm{a}}\left(2 \omega_{0}\right)-8 J_{\mathrm{c}}\left(2 \omega_{0}\right)\right] \\
R_{23}=\sqrt{3} / \sqrt{5}\left[J_{\mathrm{a}}(0)-J_{\mathrm{c}}(0)-2 J_{\mathrm{a}}\left(\omega_{0}\right)+2 J_{\mathrm{c}}\left(\omega_{0}\right)\right] \\
R_{24}=-R_{23} \\
R_{33}=\left[-J_{\mathrm{a}}(0)+J_{\mathrm{c}}(0)-2 J_{\mathrm{a}}\left(\omega_{0}\right)+2 J_{\mathrm{c}}\left(\omega_{0}\right)-2 J_{\mathrm{a}}\left(2 \omega_{0}\right)+2 J_{\mathrm{c}}\left(2 \omega_{0}\right)\right] \\
R_{34}=0
\end{gathered}
$$

and

$$
R_{44}=R_{33}
$$

Equation [9] may be solved by a numerical diagonalization method with a given initial condition, which may be obtained with the help of Eqs. [6] and [8] by following the inversion delay $\tau$ for longitudinal evolution and then the $90^{\circ}$ pulse for observation of the magnetization. The spectral lineshape is related to the real part of the Fourier-Laplace transform of the $\left(\sqrt{5} \sigma_{1}^{1}(A)+\left(\sigma_{1}^{1}\left(E_{\mathrm{a}}\right)+\sigma_{1}^{1}\left(E_{\mathrm{b}}\right)\right) / \sqrt{2}\right)$ term evolved in the acquisition period.

\section{EXPERIMENTAL}

The NMR measurements have been performed for the methyl protons of tris-acetylacetonato cobalt(III) $\left(\mathrm{Co}(\mathrm{acac})_{3}\right)$ complex in a $\mathrm{CDCl}_{3}-\mathrm{CCl}_{4}$ solution with a Bruker MSL-300 spectrometer operating at 7.05 T. $\mathrm{Co}(\mathrm{acac})_{3}$ was obtained from TCI, Tokyo Kasei, Japan, and used without further purification. The appropriate amount of $\mathrm{Co}(\mathrm{acac})_{3}$ was dissolved in $\mathrm{CDCl}_{3}(99.8 \%$, E. Merck)-CCl 4 (GR grade, E. Merck) to make a $0.05 M$ solution. The concentration of $\mathrm{CDCl}_{3}$ was $0.2 \mathrm{M}$. The sample solution was degassed with three freeze-pump-thaw cycles and then sealed in a $5 \mathrm{~mm}$ tube. The field homogeneity was carefully adjusted and the temperature was controlled at $251 \pm 0.5 \mathrm{~K}$. 


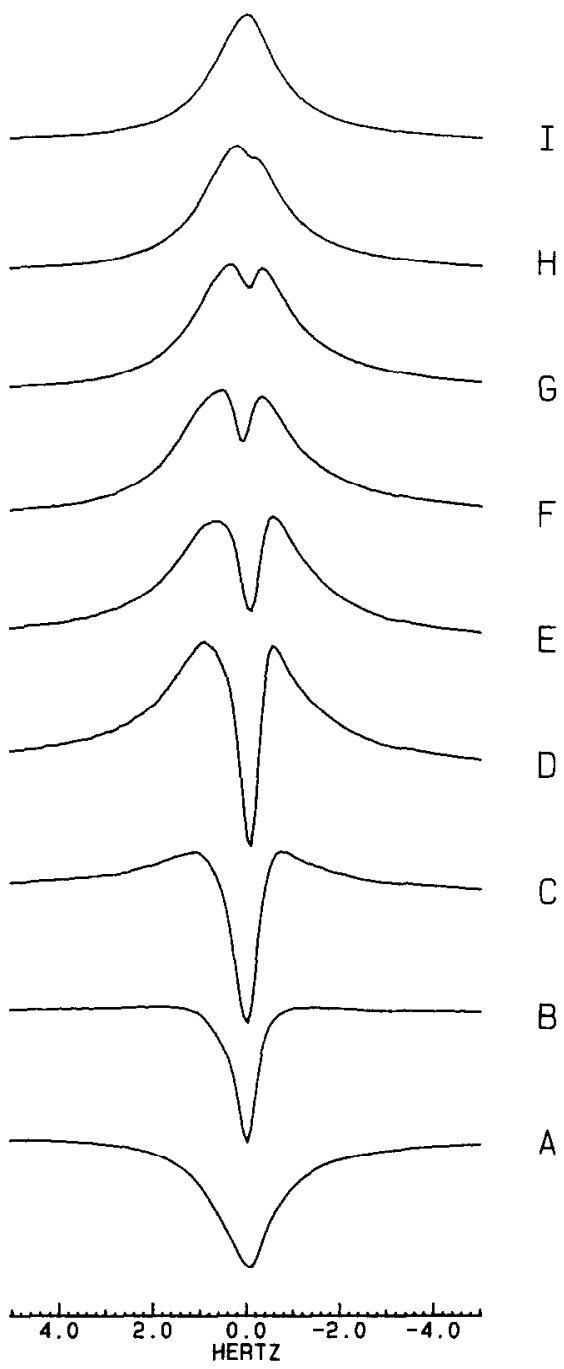

FIG. 2. Proton spectra obtained from $180^{\circ}-\tau-90^{\circ}$ experiments as a function of evolution time $\tau$ for methyl protons of $0.05 \mathrm{M} \mathrm{Co}(\mathrm{acac})_{3}$ in a $\mathrm{CDCl}_{3}-\mathrm{CCl}_{4}$ solution. The $\tau$ values and the magnifying factors for the spectral figures are given respectively by (A) $30 \mu \mathrm{s}, 1.0$; (B) $320 \mathrm{~ms}, 5.1 ;$ (C) $360 \mathrm{~ms}, 9.4$; (D) 380 ms, 13.8; (E) $440 \mathrm{~ms}, 9.8$; (F) $500 \mathrm{~ms}, 6.2$; (G) $600 \mathrm{~ms} 3.7$; (H) $900 \mathrm{~ms}, 1.9$; (I) $2.0 \mathrm{~s}$, 1.1 .

\section{RESULTS AND DISCUSSION}

The proton spectra of the methyl group obtained from the inversion recovery experiments for various $\tau$ delays in a $\mathrm{Co}(\mathrm{acac})_{3}-\mathrm{CDCl}_{3}-\mathrm{CCl}_{4}$ solution are shown in Fig. 2. The corresponding calculated spectra are displayed in Fig. 3. Magnifications of spectral intensity scales are employed in Figs. 2-4 in order to have clear comparisons. The free diffusion model is invoked for internal rotation of the methyl group, e.g., $D_{i}=(k T / I)^{1 / 2}$ where $I$ is the moment of inertia of the methyl group (16). It corresponds to $\tau_{i}=0.032 \mathrm{ps}$ at $251 \mathrm{~K}$. In the simulation, $\tau_{0}=230 \mathrm{ps}$ was used and 

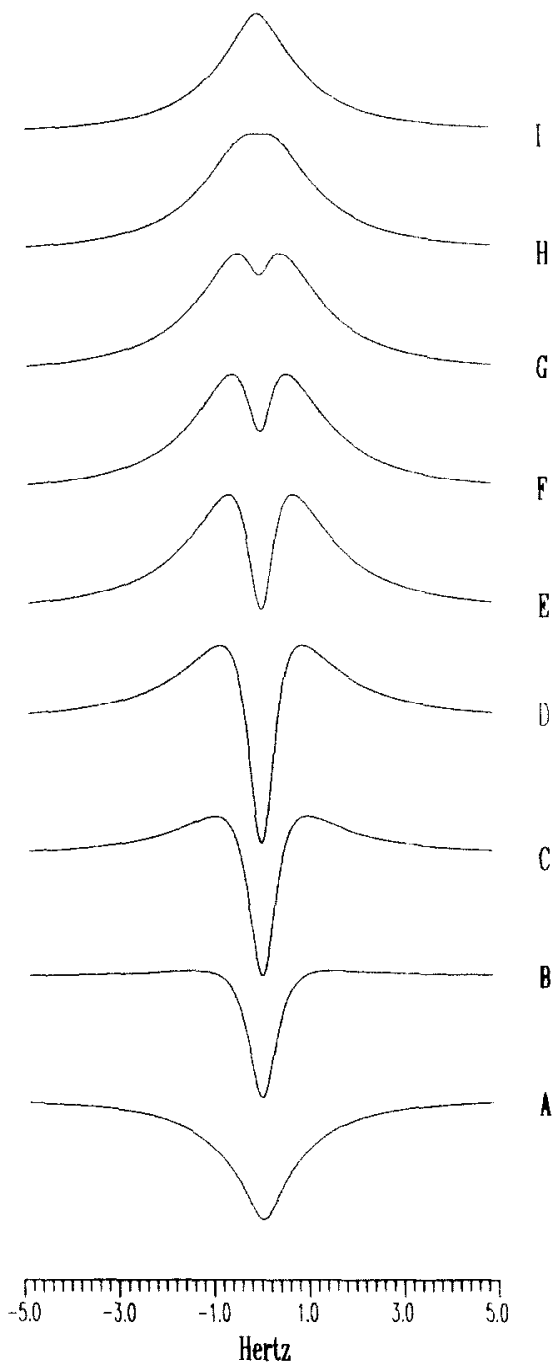

FIG. 3. Simulated proton spectra corresponding to those cited in Fig. 2 with dipole-dipole cross-relaxations included. The $\tau$ values and the magnifying factors for the spectra figures are the same as given in Fig. 2.

$0.1 \mathrm{~Hz}$ line broadening was added to take into account the unspecified transverse relaxations. Furthermore, the result is quite insensitive to the variation of $\tau_{i}$ as long as $D_{i} \gg D$ holds. In Fig. 4 the simulated spectra are obtained without the consideration of dipolc-dipole cross-interaction and it is found that the lineshapes are independent of the evolution time $\tau$ but identical to the spectrum obtained from a $90^{\circ}$ pulse experiment.

In the longitudinal relaxation, the $I_{z}$ component may be written in terms of the state multiple formalism as $\left(\sqrt{5} \sigma_{0}^{1}(A)+\left(\sigma_{0}^{1}\left(E_{\mathrm{a}}\right)+\sigma_{0}^{1}\left(E_{\mathrm{b}}\right)\right) / \sqrt{2}\right)$. Its rate equation for relaxation is given by 

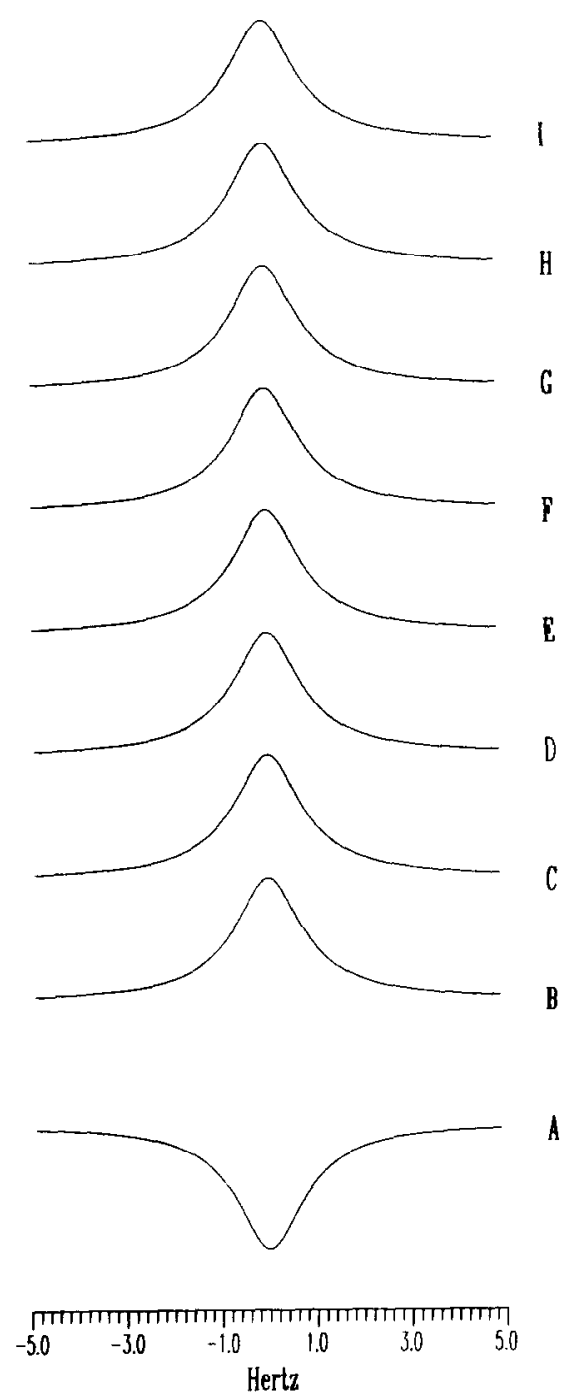

FIG. 4. Simulated proton spectra corresponding to those cited in Fig. 2 without the consideration of dipole-dipole cross-relaxations. The $\tau$ values and the magnifying factors for the spectral figures are the same as given in Fig. 2.

$$
\begin{aligned}
& d\left(\sqrt{5} \sigma_{0}^{1}(A)+\left(\sigma_{0}^{1}\left(E_{\mathrm{a}}\right)+\sigma_{0}^{1}\left(E_{\mathrm{b}}\right)\right) / \sqrt{2}\right) / d t=-\left(2 J_{\mathrm{a}}\left(\omega_{0}\right)+8 J_{\mathrm{a}}\left(2 \omega_{0}\right)\right)\left(\sqrt{5} \sigma_{0}^{1}(A)\right. \\
& \left.\quad+\left(\sigma_{0}^{1}\left(E_{\mathrm{a}}\right)+\sigma_{0}^{1}\left(E_{\mathrm{b}}\right)\right) / \sqrt{2}\right)-5\left(2 J_{\mathrm{c}}\left(\omega_{0}\right)+8 J_{\mathrm{c}}\left(2 \omega_{0}\right)\right) \sigma_{0}^{1}(A)+\left(8 J_{\mathrm{c}}\left(\omega_{0}\right)\right. \\
& \left.\left.\quad+2 J_{\mathrm{c}}\left(2 \omega_{0}\right)\right)\left(\sigma_{0}^{1}\left(E_{\mathrm{a}}\right)+\sigma_{0}^{1}\left(E_{\mathrm{b}}\right)\right) / \sqrt{2}\right)+24 / \sqrt{5}\left(-J_{\mathrm{c}}\left(\omega_{0}\right)+J_{\mathrm{c}}\left(2 \omega_{0}\right)\right) \sigma_{0}^{3}(A)
\end{aligned}
$$

This indicates that in the absence of the cross-interaction the longitudinal magnetization decays exponentially with $1 / T_{1}$ given by $\left(2 J_{\mathrm{a}}\left(\omega_{0}\right)+8 J_{\mathrm{a}}\left(2 \omega_{0}\right)\right)$. Also, it is readily shown that in extreme narrowing or in the absence of cross-relaxation the state multipoles of different rank do not couple with each other. To the contrary, the coupling between state multipoles of rank one and rank three results in the development of a 
nonuniform distribution in the longitudinal components of the state multipoles; e.g., during evolution the relative distributions among longitudinal components of the state multipoles do not follow the equilibrium-like distributions in which the state multipoles retain the relations, $\sigma_{0}^{3}(A)=0$ and the ratios $\sigma_{0}^{1}(A): \sigma_{0}^{1}\left(E_{\mathrm{a}}\right): \sigma_{0}^{1}\left(E_{\mathrm{b}}\right)$ $=\sqrt{5}: 1 / \sqrt{2}: 1 / \sqrt{2}$. At $251 \mathrm{~K}$ the $\mathrm{Co}(\mathrm{acac})_{3}$ system satisfies the motional conditions, $\omega_{0} \tau_{0} \sim 1$ and $\omega_{0} \tau_{i} \ll 1$, and therefore the contribution of dipole-dipole cross-interaction may be of importance in the relaxation process. Furthermore, in the presence of cross-interaction the dipolar coupling between quartet levels and doublet levels plays the key role in the creation of nonuniform relaxations of longitudinal elements. Also, it plays the same role in the creation of three-spin order (7).

As for the effects of dipole-dipole cross-interactions on the lineshape profiles, the most vivid difference in lineshape patterns appears around $\tau \sim 440 \mathrm{~ms}$. The cause of distinctive lineshapes is strongly correlated with the existence of the $\sigma_{0}^{3}(A)$ term (cf. Eq. [11]). It is found that $\sigma_{0}^{3}(A)$ vanishes at $\tau=0$ and $\infty$, and reaches its maximum at $\tau=470 \mathrm{~ms}$. As $\tau \gg T_{1}$ (or $\tau \ll T_{1}$ ), the longitudinal components of the state multipoles approach the (or negative temperature) equilibrium spin population. Thus, as expected, the spectra obtained with $\tau=30 \mu \mathrm{s}$ and $\tau=2 \mathrm{~s}$ yield regular lineshapes. For the case without the cross-relaxations, an earlier recovery of the inversion signal is found.

On conclusion, the variation of the peak pattern in the relaxation processes of inversion-recovery experiments demonstrates the importance of cross-correlation among the methyl protons. The spectral lineshape in longitudinal relaxation may then be utilized as a convenient method for the study of dipole-dipole cross-relaxation of methyl protons in liquids.

\section{ACKNOWLEDGMENT}

Support of this work by grants from the National Science Council of the Republic of China is gratefully acknowledged.

\section{REFERENCES}

1. L. EinARSSON AND P. O. WeSTLUND, J. Magn. Reson. 79, 54 ( 1988 ).

2. P. S. Hubbard, J. Chem. Phys. 52, 563 (1970).

3. A. WoK Aun and R. R. ERnST, Mol. Phys. 36, 317 (1978).

4. L. E. Kay And J. H. PRestegard, J. Am. Chem. Soc. 109, 3829 (1987).

5. T. C. Wong, P. L. WANG, D. M. DuH, AND L. P. Hwang, J. Phys. Chem. 93, 1295 ( 1989 ).

6. M. BÖHLEN, S. WIMPERIS, AND G. BODENHAUSEN, J. Magn. Reson. 77, 589 (1988).

7. P. L. WANG AND L. P. HWANG, J. Magn. Reson. 84, 351 ( 1989).

8. K. Blum, "Density Matrix Theory and Applications," Plenum, New York, 1981.

9. B. C. SANCTUARY, J. Magn. Reson. 61, 116 (1985).

10. I. Furó, B. Halle, AND T. C. Wong, J. Chem. Phys. 89, 5382 (1988).

11. A. Abragam, "The Principles of Nuclear Magnetism," Oxford Univ. Press, Oxford, 1961.

12. D. E. WoEsSNer, J. Chem. Phys. 37, 647 (1962).

13. D. M. BRINK AND G. R. SATChleR, “Angular Momentum,” Oxford Univ. Press, Oxford, 1968.

14. A. G. REDFTELD, in "Advances in Magnetic Resonance" (J. S. Waugh, Ed.), Vol. 1, p. 1, Academic Press, New York, 1965.

15. L. G. WERBELOW AND D. M. Grant, in "Advances in Magnetic Resonance" (J. S. Waugh, Ed.), Vol. 9. p. 189, Academic Press, New York, 1977.

16. J. B. Lambert, R. J. Nienhuis, AND J. W. Keepers, Angew. Chem. Int. Ed Eng. 20, 487 ( 1981 ). 